\title{
Evaluación Poblacional del Camarón Cryphiops caementarius en Ríos de la Costa Sur del Perú
}

\author{
Population Assessment of Prawn Cryphiops caementarius on Rivers of the \\ South Coast of Peru
}

\author{
José Wasiw G. ${ }^{1,2}$, Víctor Yépez P.,3
}

\section{Resumen}

Se reportan aspectos biológicos pesqueros y poblacionales del «camarón de río» Cryphiops caementarius con base a prospecciones efectuadas en los ríos Ocoña, MajesCamaná y Tambo, en el periodo setiembre-diciembre de 2013. Se observó la presencia de una fracción mínima de ejemplares con tallas superiores a los $120 \mathrm{~mm}$, reportándose las mayores tallas en el río Majes-Camaná (moda: $79 \mathrm{~mm}$ ). Se encontró una mayor proporción de hembras en el río Majes-Camaná, predominancia de gónadas en el estadio de maduración incipiente en los ejemplares de los ríos Ocoña y Tambo, mayor densidad (individuos $\left./ \mathrm{m}^{2}\right)$ y biomasa media $\left(\mathrm{g} / \mathrm{m}^{2}\right)$ en los estratos altitudinales inferiores y mayores índices de concentración del recurso en los ríos Majes-Camaná y Tambo. Asimismo, los principales parámetros fisicoquímicos de calidad del agua evidenciaron condiciones favorables para el desarrollo poblacional del recurso.

Palabras clave: camarón de río, pesca ilegal, relación longitud-peso, densidad, biomasa

\section{Abstract}

Fisheries and biological aspects of the freshwater prawn Cryphiops caementarius are reported based on surveys in the Ocoña, Majes-Camana, and Tambo rivers in the period September-December 2013. A small fraction of the population showed sizes larger than $120 \mathrm{~mm}$, where the largest individuals were found in the Majes-Camana river (mode: $79 \mathrm{~mm}$ ). There were a higher proportion of females in the Majes-Camana river, predominance of gonads in the incipient stage of maduration in individuals of the Ocoña

${ }^{1}$ Área Funcional de Investigaciones de Recursos en Aguas Continentales, Instituto del Mar del Perú, Callao, Perú

${ }^{2}$ E-mail:jwasiw@imarpe.gob.pe

${ }^{3}$ E-mail:vyepez@imarpe.gob.pe

Recibido: 30 de mayo de 2014

Aceptado para publicación: 25 de enero de 2015 
and Tambo rivers, higher density (individuals $/ \mathrm{m}^{2}$ ) and higher biomass $\left(\mathrm{g} / \mathrm{m}^{2}\right)$ in the lower altitudinal strata, and larger concentration rates in the Majes-Camana and Tambo rivers. Besides, the main physicochemical parameters of water quality showed favorable conditions for the prawn population development.

Key words: fresh water prawn, illegal fishing, length-weight relationship, density, biomass

\section{INTRODUCCIÓN}

El camarón de río, principal recurso hidrobiológico de los cuerpos de agua de la vertiente occidental de los Andes del Perú, involucra a los géneros Cryphiops, Macrobrachium, Palaemon y Atya; siendo Cryphiops caementarius la especie mayoritaria (o más abundante), base de una importante pesquería artesanal en los ríos de la costa sur del Perú y norte de Chile, entre los $6^{\circ} 30^{\prime}$ y $33^{\circ} \mathrm{LS}$ (Bahamonde y Vila, 1971; Amaya y Guerra, 1976).

El camarón de río C. caementarius, especie endémica de los ríos de la vertiente occidental de los Andes, habita los cuerpos de agua lóticos costeros del Perú al sur del río Chancay-Lambayeque y los del litoral norte chileno hasta los $33^{\circ} \mathrm{S}$ (Bahamonde y Vila, 1971). Se dispone de reportes que señalan una distribucional altitudinal hasta los 1400 msnm (Báez, 1985), dependiendo de la pendiente o grado de desnivel del lecho del río; asimismo, se estima que aproximadamente el $80 \%$ del recurso existente en la costa peruana se distribuye en los ríos de Arequipa, principalmente Ocoña, Majes-Camaná y Tambo (Yépez, 2009), en los cuales su explotación es de notoria importancia.

Los ejemplares adultos de mayor tamaño permanecen en los estratos altitudinales superiores durante gran parte del año. Machos maduros e inmaduros, ejemplares juveniles y hembras inmaduras presentan una mayor abundancia en zonas medias y altas de las cuencas de los ríos, mientras que las hembras maduras y aquellas que portan huevos se distribuyen mayormente en las zonas bajas del río, concentrándose en los últimos 20-30 km y en zonas cercanas a la desembocadura del río, donde se produce la eclosión de los huevos. En cuanto a la distribución diferencial transversal (ancho del río), los ejemplares adultos se encuentran en zonas de mayor profundidad y los juveniles en zonas someras (mayoritariamente en orillas o bordes del río) (Castro, 1966).

La primera parte de la vida larvaria transcurre en los ambientes de estuario (zona de mezcla entre las aguas de mar y de río). El recurso desova durante todo el año, con máxima actividad reproductiva entre los meses de diciembre y marzo coincidente con la estación de avenidas («aguas turbias»), periodo en el que hay veda para su captura (R.M. N. ${ }^{\circ}$ 312-2006-PRODUCE). Tiene la cualidad de refugiarse en el interior de cuevas que construye con limo, entre las piedras y huecos, dejando sobresalir las antenas durante el día. Esta especie no habita cursos de agua con temperatura menor de $10^{\circ} \mathrm{C}$ (Castro, 1966).

Existe una amplia información sobre aspectos bioecológicos y reproductivos de $C$. caementarius (Ancieta, 1950; Chávez et al., 1973; Elías, 1960; Hartmann, 1958; Norambuena, 1977; Viacava et al., 1978), siendo menor la información relacionada al ciclo de vida y desarrollo larvario (Munaylla, 1977; Vegas et al., 1981; Rivera et al., 1983; Sanzana y Báez, 1983; Meruane et al., 2006; Yavar y Dupré, 2007). 
El análisis de la serie histórica de los índices de concentración de C. caementarius en los cauces de los ríos de la región Arequipa muestra un significativo descenso de estos, especialmente por la explotación clandestina con extracción ilícita del recurso, además de las alteraciones del hábitat por procesos naturales de sequía y antrópicos relacionados con el uso del agua y lecho de los ríos, entre ellos la construcción de estructuras hidráulicas, construcción de carreteras y desarrollo de actividades agrícolas y mineras. Esto ha provocado la reducción del tamaño de las poblaciones de este crustáceo y la disminución de la proporción de ejemplares con tallas superiores a los $70 \mathrm{~mm}$ (talla mínima de captura comercial).

La norma R.M. N. ${ }^{\circ}$ 312-2006-PRODUCE establece medidas referidas al periodo de veda definida del recurso, la que está orientada únicamente a la protección de una fracción de la población (stocks comerciales y precomerciales en el periodo de máxima actividad reproductiva), no habiéndose establecido cuotas de captura. Estudios realizados en Chile (Meruane et al., 2006) muestran que la «crianza» (engorde) de la especie a escala comercial contribuiría a la producción sostenible y bajaría la presión sobre sus stocks.

En el Perú, el Instituto del Mar del Perú viene ejecutando actividades de monitoreo poblacional a fin de aportar bases consistentes para el manejo de su pesquería. Ello comprende la realización de prospecciones anuales en los ríos de la costa centro-sur, dirigidas principalmente a determinar índices de concentración y estimar niveles de abundancia de los stocks comerciales y precomerciales en los ríos donde existe extracción intensa del recurso. Los monitoreos poblacionales se han centralizado en los ríos de la región Arequipa, debido a la importancia de la actividad extractiva que en ellos se ejecuta. El presente estudio muestra los resultados de las prospecciones poblacionales de camarón ejecutadas en el periodo setiembre-diciembre de 2013, específicamente en los ríos Tambo, Ocoña y Majes-Camaná.

\section{Materiales y Métodos}

\section{Esquema de Muestreo}

El trabajo comprendió los ríos Tambo, Ocoña y Majes-Camaná en la región Arequipa, al sur del Perú. Los cauces de los ríos fueron divididos en estratos de 100 metros de altitud, según el esquema de estratificación establecido en las prospecciones iniciales del programa de monitoreo. Asimismo, la selección se hizo por cartografía y comprobación in situ de las condiciones de acceso a cada área definida como estación para pesca exploratoria.

Para determinar el área de sección mojada o espejo de agua de cada estrato, elemento base para las estimaciones poblacionales, se consideró la longitud del curso (definido por cartografía) y en el terreno se definió el ancho promedio del lecho; ajustándose ambas magnitudes por coeficientes referidos a factores de sinuosidad (meandro) y morfometría dendrítica (ramal o brazo).

Los trabajos de campo (análisis de parámetros fisicoquímicos y faenas de pesca) se ejecutaron en el área comprendida en los siguientes rangos altitudinales: río Ocoña 0-600 msnm (24 estaciones de muestreo), río Majes-Camaná 0-1000 msnm (31 estaciones de muestreo) y río Tambo 0-400 msnm (16 estaciones de muestreo). Se tuvo entre 3 y 5 estaciones por estrato altitudinal. Los detalles relacionados a la estratificación de los cauces y la ubicación de las estaciones de evaluación se muestran en la Fig. 1. 

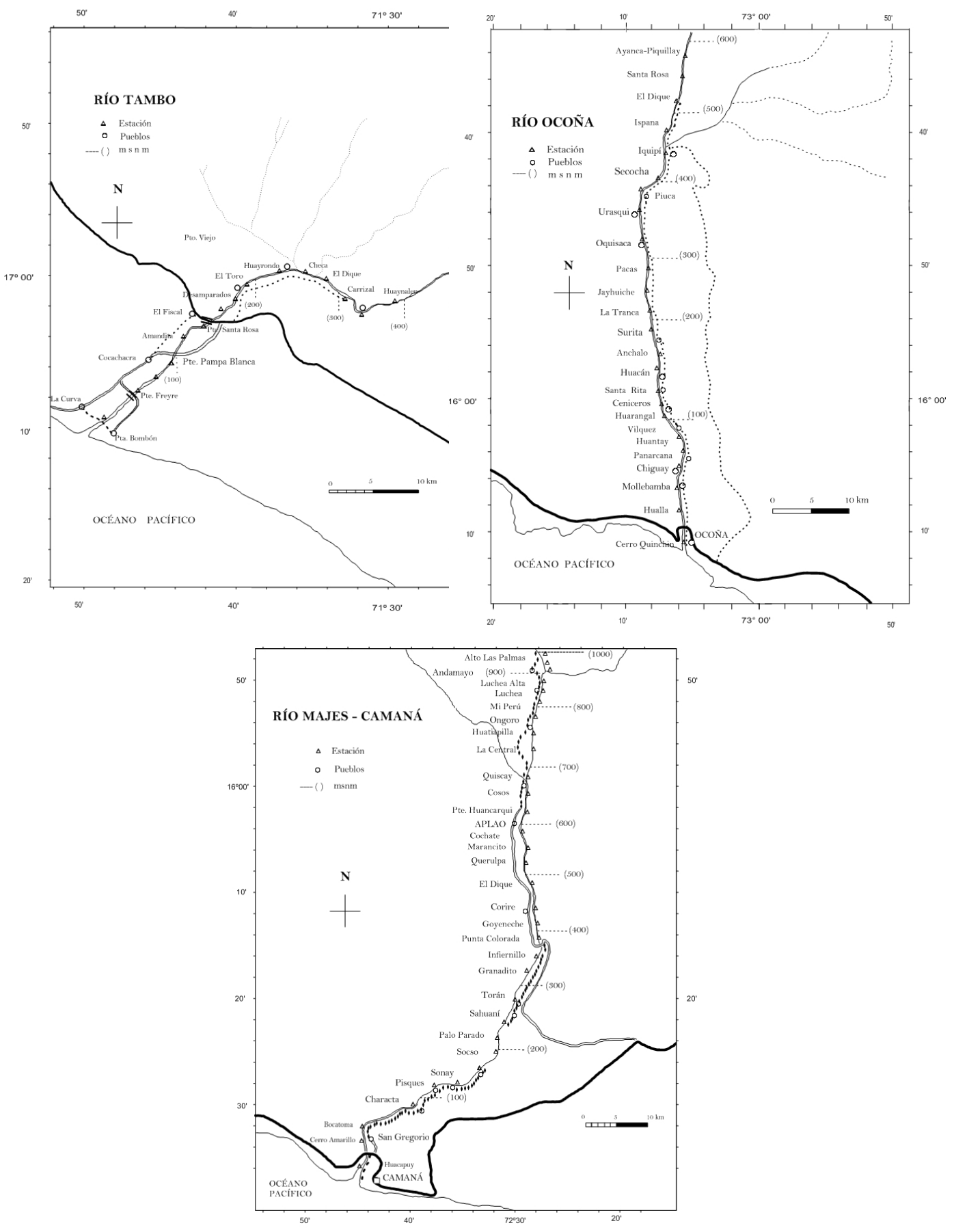

Figura 1. Estaciones de muestreo para evaluación de «camarón de río» (Cryphiops caementarius) en los ríos Tambo (septiembre 2013), Ocoña (octubre-noviembre 2013), y Majes-Camaná (diciembre 2013) 


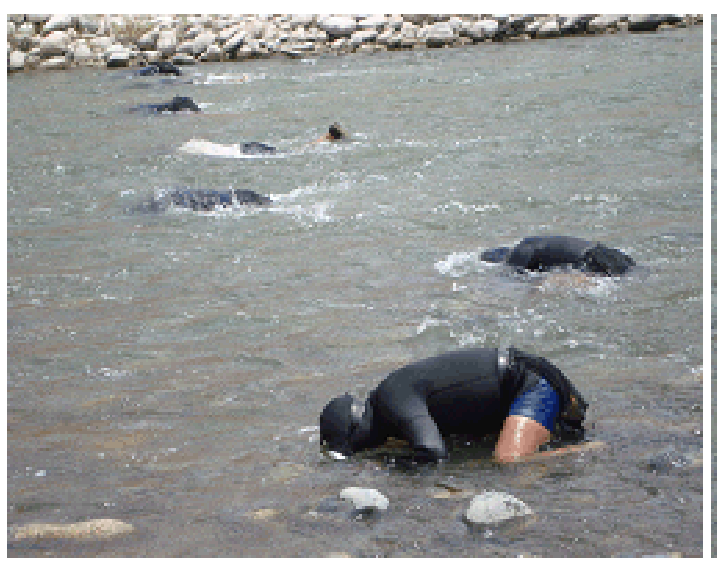

A

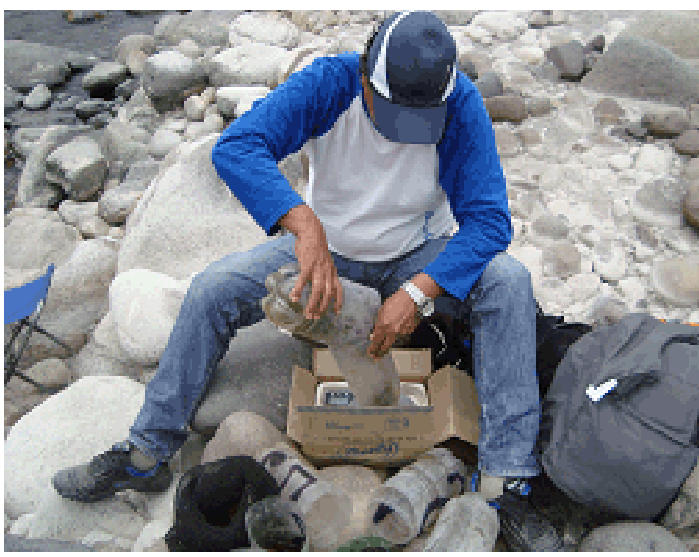

C

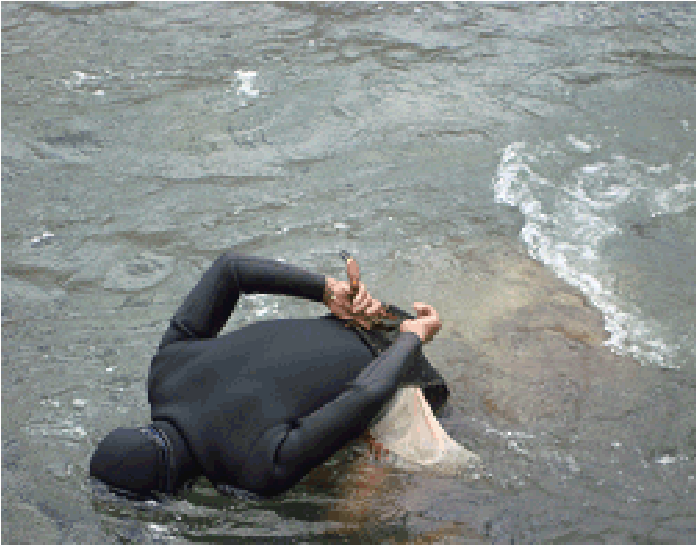

B

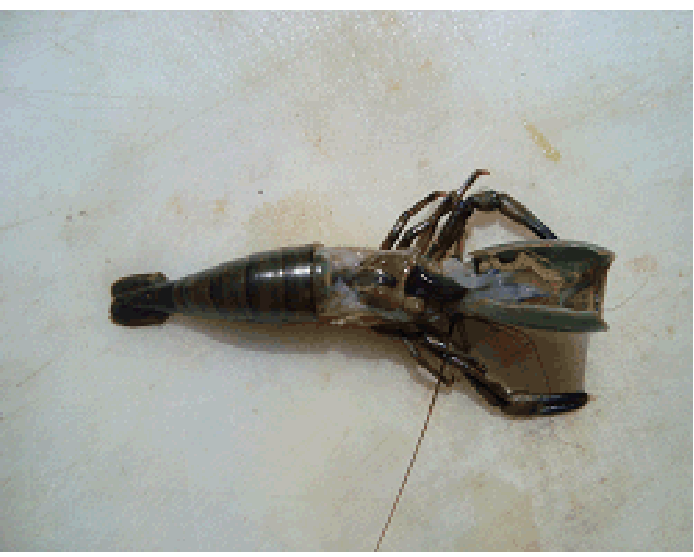

D

Figura 2. Recolección del camarón. A: «Barrido» para la captura; B: Colección del camarón en el «chinguillo»; C: Pesaje de la captura de camarón; D: Hembra grávida

\section{Características Abióticas del Medio Hídrico}

Se determinaron los parámetros fisicoquímicos de calidad de agua por estación. Se midió la temperatura del agua (con termómetro protegido, precisión de $0.1^{\circ} \mathrm{C}$ ), temperatura ambiente, $\mathrm{pH}$ (pHmetro digital), oxígeno disuelto y porcentaje de saturación (oxímetro YSI), así como $\mathrm{CO}_{2}$ libre, dureza, alcalinidad total y fenolftaleína, cloruros disueltos y $\mathrm{ClNa}$, y nitratos y nitritos mediante kits comerciales específicos (Hach, EEUU).

Las muestras de agua fueron obtenidas a nivel superficial en la zona de orilla, realizándose los análisis in situ. Asimismo, se registraron observaciones acerca de la presen- cia de brazos, sinuosidades o meandros, además del color del agua en cada una de las estaciones de muestreo.

\section{Faenas de Pesca Exploratoria}

Las prospecciones se ejecutaron en las secciones media y baja de las cuencas de los ríos, donde la disponibilidad de los stocks comerciales y precomerciales es mayor.

Las capturas del recurso se realizaron mediante el método del «buceo a pulmón» (Fig. 2).

La operación de pesca consideró un «barrido» en contracorriente, efectuado por un equipo de ocho extractores experimenta- 
dos, quienes recorrieron como término medio transectos de $40 \mathrm{~m}$ de largo en dos entradas, en un periodo aproximado de 20 minutos (dependiendo del tipo de fondo y caudal del río).

Se hizo el conteo y pesaje (en gramos) de los especímenes obtenidos por transecto. Asimismo, se registró el número de ejemplares que escaparon, según información verbal de cada extractor, a fin de estimar factores de eficiencia en la operación de pesca e índices de abundancia.

\section{Muestreo Biométrico y Biológico}

De las capturas obtenidas por estación, se separó al azar una muestra de 60 especímenes y, en los casos en que la captura fuera menor, se trabajó con el total de individuos. Los camarones fueron pesados con balanza electrónica portátil de $0.1 \mathrm{~g}$ de precisión y medidos con un vernier mecánico (en $\mathrm{mm}$ ) y agrupados en clases de $5 \mathrm{~mm}$ a la unidad inferior.

Se registró longitud total ( $\mathrm{mm})$, longitud del cefalotórax $(\mathrm{mm})$, peso total $(\mathrm{g})$, peso abdominal (g), sexo y madurez gonadal. Asimismo, se registró la falta de apéndices, color, estado reproductivo (ovígera o no) y estado de la muestra. Los parámetros para la determinación de la madurez gonadal fueron tomados de Viacava et al. (1978).

\section{Aspectos Poblacionales}

En la estimación de la biomasa se empleó una modificación del método por «área barrida», que consiste en calcular la densidad de la especie objetivo, relacionando las capturas con el área o sector de barrido efectivo y extrapolándola a la totalidad del área de cada estrato. Para el efecto, se siguieron los procedimientos descritos por Espino y Wosnitza-Mendo (1984), mediante los cuales se emplea el número y peso de ejemplares capturados por extractor (considerando los escapes) en cada sección. Se estimaron índices de concentración en número y biomasa por estratos y ríos, los cuales fueron comparados con los estimados obtenidos en periodos previos.

\section{Resultados y Discusión}

\section{Parámetros Fisicoquímicos de Calidad del Agua}

En el Cuadro 1 se observa los valores mínimos y máximos de los parámetros fisicoquímicos registrados en las prospecciones en los tres ríos. Se considera como cursos de agua con condiciones aparentes para el desarrollo del camarón de río, aquellos con valores dentro de los siguientes rangos: temperatura 15 a $27{ }^{\circ} \mathrm{C}, \mathrm{pH} 6.5$ a 9.0, oxígeno disuelto 5 a $10 \mathrm{mg} / \mathrm{L}$, alcalinidad total 100 a $200 \mathrm{mg} / \mathrm{L}$ y dureza total 150 a $400 \mathrm{mg} / 1 \mathrm{de}$ $\mathrm{CaCO}_{3}$ (Bahamonde y Vila, 1971; Chávez et al., 1973; Norambuena, 1977; Viacava et al., 1978; Meruane et al., 2006). Los resultados evidenciaron condiciones favorables para el desarrollo poblacional del camarón de río $C$. caementarius.

En el cauce del río Tambo se encontraron significativos valores de la alcalinidad (alta productividad) en zonas ribereñas próximas a centros poblados, así como de cloruros y dureza, especialmente en la parte media del cauce (poblados de Len y Carrizal), la parte baja (Cocachacra, Puente Freyre y La Curva) y en el estuario del río, donde existe una mayor carga orgánica.

En el río Ocoña se apreciaron incrementos en la concentración de oxígeno disuelto, $\mathrm{CO}_{2}$, dureza y $\mathrm{pH}$ en los lugares próximos a zonas urbanas (Iquipí, Oquisaca y Panarcana), donde se aprecia el mayor ingreso de desechos domésticos al cauce del río. Asimismo, se pudo observar un incremento importante y progresivo de la actividad minera informal, específicamente en el sector del poblado de Secocha. 
En el río Majes-Camaná existen condiciones aparentes para el desarrollo del camarón. El incremento en los valores de dureza probablemente se relacione con la intensa actividad agrícola que se desarrolla en el valle de Majes. Los mayores valores de concentración de oxígeno disuelto, $\mathrm{CO}_{2}$ y cloruros se relacionan con la cercanía a áreas de cultivo y poblados en la zona próxima a la desembocadura del río.

En líneas generales, las condiciones de calidad de agua son aparentes para el desarrollo poblacional del camarón de río $C$. caementarius; sin embargo, algunas alteraciones de los valores de los parámetros fisicoquímicos con respecto al rango considerado «normal» para la presencia del recurso tendrían relación con el ingreso de aguas servidas (sin tratamiento alguno), así como por el vertimiento de relaves mineros al cauce del río o por su ingreso por escorrentía de aguas pluviales. Otras alteraciones pueden ser causadas por la construcción de estruc- turas físicas en el cauce de los ríos, por el uso de pesticidas con fines agrícolas y por envenenamiento de las aguas como método de pesca ilegal.

\section{Aspectos Biológicos}

\section{Composición por tallas}

Las tallas encontradas en el camarón en los tres ríos en estudio se muestran en el Cuadro 2, habiendo diferencias marcadas entre las tallas mínimas y máximas de hembras y machos de los tres ríos.

Las modas de la tallas del camarón de río en los primeros estratos altitudinales inferiores de las tres cuencas estuvieron por debajo de la talla comercial. Por otro lado, la distribución porcentual en los ríos Ocoña y Majes-Camaná se aproximó a una distribución normal, siendo dominante la fracción de ejemplares con tallas iguales y superiores a los $70 \mathrm{~mm}$ (57.1 y $63.7 \%$, respectivamente),

Cuadro 1. Valores mínimos y máximos de los parámetros fisicoquímicos del agua registrados en las prospecciones del año 2013 en los ríos Tambo, Ocoña y MajesCamaná

\begin{tabular}{lccc}
\hline Parámetros fisicoquímicos & Tambo & Ocoña & Majes-Camaná \\
\hline Altitud $(\mathrm{msnm})$ & $0-400$ & $0-600$ & $0-1000$ \\
Temperatura del agua $\left({ }^{\circ} \mathrm{C}\right)$ & $19.1-25.7$ & $18.4-26.9$ & $20.4-26.8$ \\
Temperatura ambiental $\left({ }^{\circ} \mathrm{C}\right)$ & $18.8-24.2$ & $18.1-25.2$ & $21.3-30.0$ \\
$\mathrm{pH}$ & $8.7-9.1$ & $8.9-10.1$ & - \\
Oxígeno disuelto $(\mathrm{mg} / \mathrm{L})$ & $6.3-11.6$ & $6.1-10.4$ & $6.7-11.2$ \\
Oxígeno disuelto $(\% \mathrm{sat})$. & $72-133$ & $67-123$ & $82-134$ \\
$\mathrm{CO}_{2}$ libre & $8-16$ & $8-14$ & $8-18$ \\
Dureza CaCO $(\mathrm{mg} / \mathrm{L})$ & $343-462$ & $86-154$ & $171-325$ \\
Alcalinidad fenolf. $(\mathrm{mg} / \mathrm{L})$ & 0 & 0 & - \\
Alcalinidad total $(\mathrm{mg} / \mathrm{L})$ & $132-224$ & $92-108$ & - \\
Cloruro CL $(\mathrm{mg} / \mathrm{L})$ & $80-140$ & $80-160$ & $129-180$ \\
Cloruro CLNa $(\mathrm{mg} / \mathrm{L})$ & $128-224$ & $128-256$ & $192-288$ \\
\hline
\end{tabular}


Cuadro 2. Valores mínimos y máximos de longitud total $(\mathrm{mm})$, según sexo, de camarón Cryphiops caementarius en ríos de la costa sur (setiembre-diciembre, 2013)

\begin{tabular}{lccccccccc}
\hline Sexo & \multicolumn{3}{c}{ Tambo } & \multicolumn{3}{c}{ Ocoña } & \multicolumn{3}{c}{ Majes-Camaná } \\
\cline { 2 - 10 } & Moda & Máx. & Mín. & Moda & Máx. & Mín. & Moda & Máx. & Mín. \\
\hline Hembra & 69 & 101 & 35 & 70 & 99 & 33 & 79 & 107 & 41 \\
Macho & 69 & 121 & 41 & 65 & 138 & 37 & 71 & 150 & 45 \\
\hline Total & 69 & 121 & 35 & 71 & 138 & 37 & 79 & 150 & 41 \\
\hline
\end{tabular}

Cuadro 3. Valores mínimos y máximos de longitud total (LT en mm) de camarón Cryphiops caementarius, en ríos de la costa sur $^{1}$

\begin{tabular}{cccccccccc}
\hline \multirow{2}{*}{ Años } & \multicolumn{3}{c}{ Río Ocoña } & \multicolumn{3}{c}{ Río Majes-Camaná } & \multicolumn{3}{c}{ Río Tambo } \\
\cline { 2 - 9 } & moda & LT mín & LT máx & moda & LT mín & LT máx & moda & LT mín & LT máx \\
\hline 2005 & 66 & 40 & 132 & 67 & 37 & 146 & 55 & 26 & 132 \\
2006 & 65 & 34 & 128 & 67 & 30 & 163 & 57 & 21 & 114 \\
2007 & 60 & 35 & 128 & 67 & 41 & 148 & 57 & 34 & 118 \\
2008 & 71 & 38 & 134 & 68 & 34 & 122 & 63 & 33 & 127 \\
2009 & 67 & 40 & 127 & 68 & 32 & 154 & 78 & 27 & 132 \\
2010 & 72 & 30 & 141 & 65 & 41 & 138 & 66 & 28 & 129 \\
2011 & 72 & 38 & 149 & 73 & 34 & 142 & 73 & 26 & 140 \\
2012 & 74 & 36 & 139 & 68 & 34 & 134 & 66 & 32 & 144 \\
2013 & 71 & 37 & 138 & 79 & 41 & 150 & 69 & 35 & 121 \\
\hline
\end{tabular}

${ }^{1}$ Fuente: Prospecciones de evalua ción poblacional del cama rón de río ejecutadas por IMARPE

mientras que en el río Tambo el $52.2 \%$ de ejemplares presentaron tallas inferiores a 70 mm(Fig. 3). Datos históricos del 2005 al 2013 indican que las mayores tallas se registraron en el río Majes-Camaná, en tanto que las menores tallas se observaron en el río Tambo (Cuadro 3).

En general, hay una leve tendencia al decremento de las tallas, lo cual podría ser consecuencia de varios factores; entre ellos pueden considerarse como los principales la intensa actividad extractiva dirigida a ejemplares de mayores tallas (debido a que su comer-cialización reporta mayor beneficio económico), así como el incremento de la accesibilidad a zonas de pesca.

Por ello, además, en los últimos años se habría incrementado la probabilidad de ocurrencia de capturas de ejemplares con tallas inferiores a los $70 \mathrm{~mm}$ en las cuencas prospectadas, al igual que el esfuerzo de pesca y el empleo de artes y métodos ilícitos de pesca. El mayor porcentaje de capturas no lícitas correspondería al sector inferior de las cuencas. 
Río Tambo (Setiembre 2013)
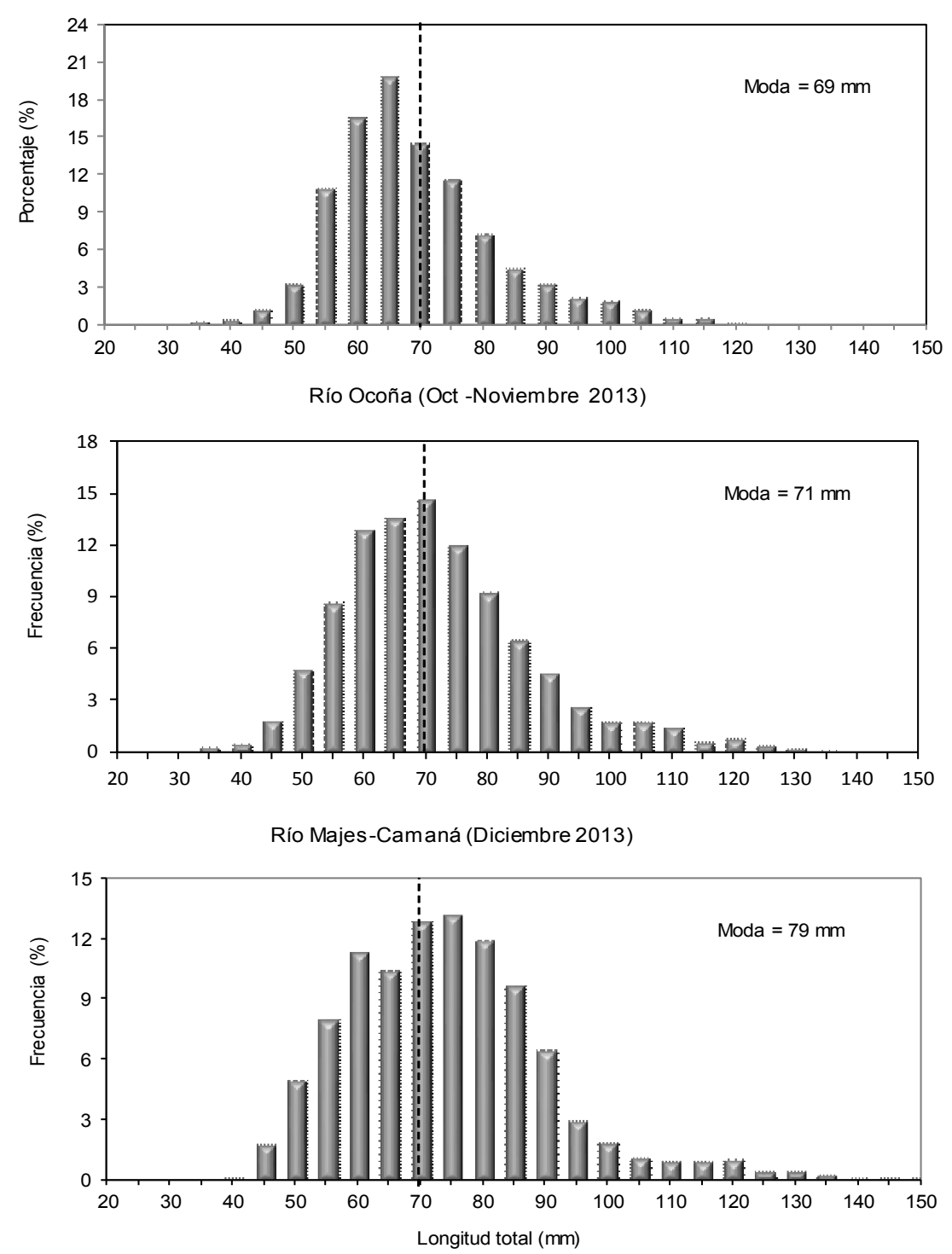

Figura 3. Distribución porcentual de las tallas del camarón Cryphiops caementarius en ríos de la costa sur del Perú (setiembre - diciembre 2013)

Proporción de sexos

Los camarones machos predominan sobre las hembras en todos los estratos altitudinales de los ríos Ocoña y Tambo, en tanto que las hembras predominaron en el río
Majes-Camaná (Cuadro 4). No obstante, el patrón natural de distribución altitudinal de la especie corresponde a un predominio de hembras en los estratos altitudinales inferiores y de machos en los superiores (Viacava et al., 1978). 

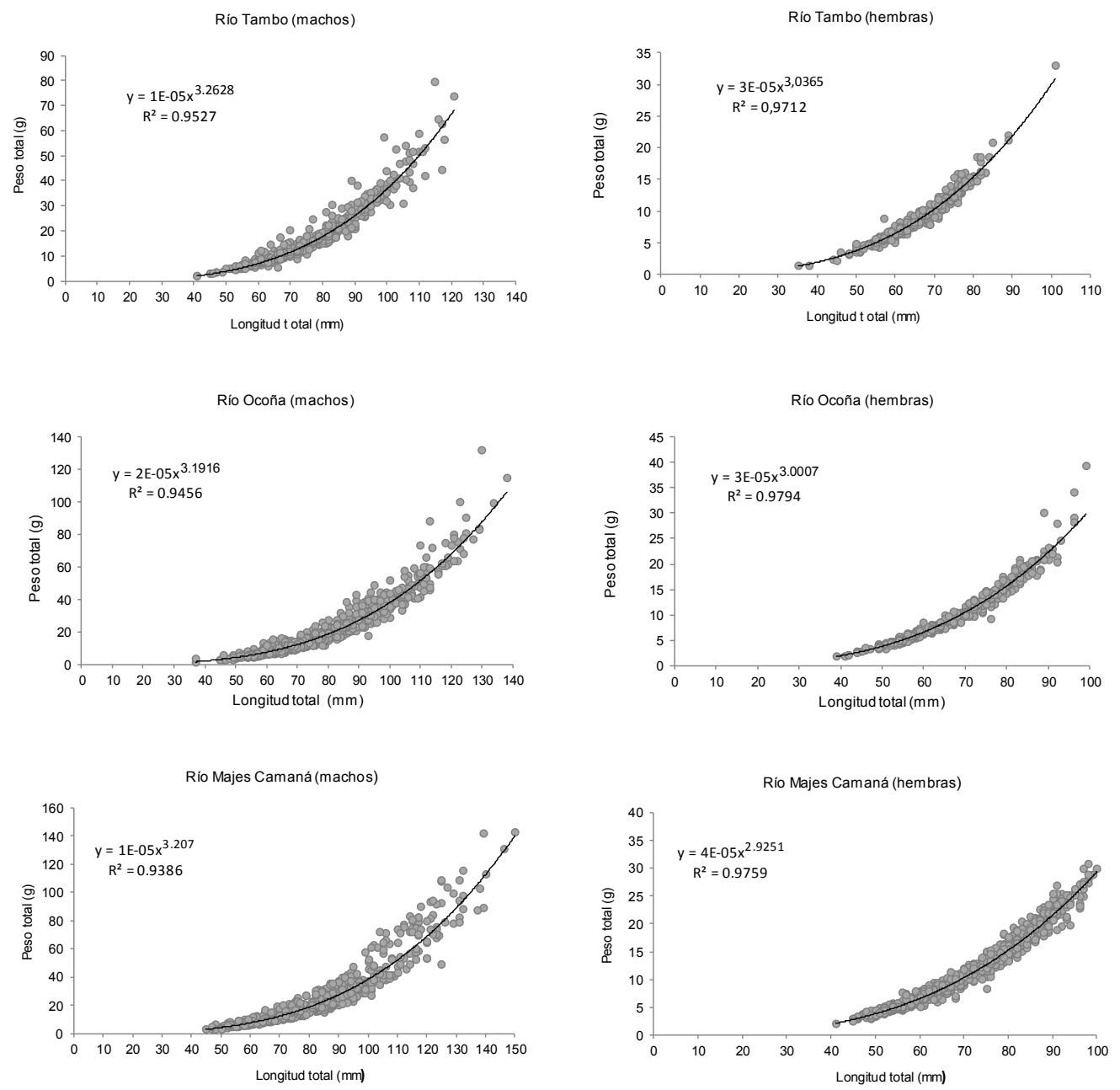

Figura 4. Relación longitud total - peso total, según sexo, en el camarón Cryphiops caementarius en ríos de la costa sur (setiembre-diciembre 2013)

\section{Relación longitud - peso}

El análisis de la relación longitud-peso indicó que las poblaciones de camarón de río están compuestas por ejemplares con mayor peso respecto a su talla en los tres ríos evaluados, siendo más notorio en los ejemplares machos (Fig. 4).

La relación existente entre las variables peso total - peso abdominal en camarones mayores de $7 \mathrm{~cm}$ fue lineal y estadísticamente significativa, según el análisis de regresión. Su análisis permite conocer el valor de peso total necesario para obtener un «peso comercial neto» (compuesto tan solo por colas). $\mathrm{Al}$ respecto, los individuos capturados en el río Majes-Camaná presentaron un menor rendimiento en peso para colas (para el total de la muestra analizada, así como para los ejemplares con tallas mayores de $70 \mathrm{~mm}$ ), lo que se explica por la presencia de machos de tallas mayores, donde el mayor porcentaje del peso está representado en las quelas y el cefalotórax. 
Cuadro 4. Proporción de sexos (macho / hembra) de camarón Cryphiops caementarius por estratos altitudinales en ríos de la costa sur (setiembre-diciembre 2013)

\begin{tabular}{llll}
\hline Estrato & Ocoña & $\begin{array}{c}\text { Majes- } \\
\text { Camaná }\end{array}$ & Tambo \\
\hline $901-$ & & $0.5 / 1.0$ & \\
1000 & & $0.7 / 1.0$ & \\
$801-900$ & & $0.6 / 1.0$ & \\
$701-800$ & & $0.6 / 1.0$ & \\
$601-700$ & & $0.7 / 1.0$ & \\
$501-600$ & $1.7 / 1.0$ & & \\
$401-500$ & $1.0 / 1.0$ & $0.5 / 1.0$ & \\
$301-400$ & $1.2 / 1.0$ & $1.0 / 1.0$ & $1.6 / 1.0$ \\
$201-400$ & $1.1 / 1.0$ & $0.7 / 1.0$ & $0.7 / 1.0$ \\
$101-200$ & $1.7 / 1.0$ & $0.6 / 1.0$ & $1.2 / 1.0$ \\
$0-100$ & $1.4 / 1.0$ & $0.8 / 1.0$ & $1.0 / 1.0$ \\
\hline Total & $1.3 / 1.0$ & $0.6 / 1.0$ & $1.1 / 1.0$ \\
\hline
\end{tabular}

Estructura de la madurez gonadal

Se observó una alta predominancia de gónadas en estadio II, o de maduración incipiente, en los ríos Ocoña y Tambo (mayor de $85 \%$, tanto de hembras como de machos), mientras que en el río Majes-Camaná fue de $53.0 \%$ de machos y $37.1 \%$ de hembras (Cua- dro 5). En este río, se observó, asimismo, que el 51.8 y $11.2 \%$ de ejemplares hembras presentaban sus gónadas en estadios III y IV, respectivamente, valores mayores que los observados en años previos, lo cual implicaría una proximidad del pico de desove a ocurrir en el periodo de verano donde se veda el recurso (R.M. N. ${ }^{\circ}$ 312-2006-PRODUCE).

\section{Aspectos Poblacionales de los Adultos}

\section{Índices de concentración}

El promedio ponderado de densidad varió entre 0.49 y $1.34 \mathrm{indiv} / \mathrm{m}^{2}$, mientras que el de biomasa media varió entre 6.32 y $16.70 \mathrm{~g} /$ $\mathrm{m}^{2}$; donde los menores valores se registraron en los estratos altitudinales del río Ocoña y los mayores en el río Majes-Camaná (Cuadro 6). Asimismo, estos valores muestran la tendencia decreciente que se observa en el río Ocoña, según datos históricos de IMARPE (Cuadro 7).

Las fluctuaciones en los índices de concentración según estrato altitudinal se relacionan con el grado de accesibilidad a las zonas de pesca, intensidad de la actividad extractiva y desarrollo de diversas actividades que tienen influencia directa sobre el recurso hídrico (incluyendo la construcción de estructuras de barrera en el curso del río y desvío de las aguas) y, por tanto, en las poblaciones del camarón.

Cuadro 5. Estadios de madurez gonadal (\%), según estadios sexuales, del camarón Cryphiops caementarius en ríos de la costa sur (setiembre-diciembre, 2013)

\begin{tabular}{ccccccc}
\hline \multirow{2}{*}{$\begin{array}{c}\text { Estadio de } \\
\text { madurez }\end{array}$} & \multicolumn{2}{c}{ Río Ocoña } & \multicolumn{2}{c}{ Río Majes-Camaná } & \multicolumn{2}{c}{ Río Tambo } \\
\cline { 2 - 7 } & Macho & Hembra & Macho & Hembra & Macho & Hembra \\
\hline I & 0.1 & 0.2 & 0 & 0 & 0.2 & 0.2 \\
II & 95.3 & 87.8 & 53.0 & 37.1 & 95.4 & 86.1 \\
III & 3.9 & 10.8 & 46.7 & 51.8 & 3.1 & 13.5 \\
IV & 0.6 & 1.2 & 0.3 & 11.2 & 2.3 & 0.2 \\
V & 0 & 0 & 0 & 0 & 0 & 0 \\
\hline
\end{tabular}


Cuadro 6. Densidad (individuos $\left./ \mathrm{m}^{2}\right)$ y biomasa media $\left(\mathrm{g} / \mathrm{m}^{2}\right)$ de camarón Cryphiops caementarius, según estratos altitudinales en los ríos de la costa sur (2013) ${ }^{1}$

\begin{tabular}{lcccccc}
\hline \multirow{2}{*}{ Estratos } & \multicolumn{2}{c}{ Río Ocoña } & \multicolumn{2}{c}{ Río Majes-Camaná } & \multicolumn{2}{c}{ Río Tambo } \\
\cline { 2 - 7 } & Indiv $/ \mathrm{m}^{2}$ & $\mathrm{~g} / \mathrm{m}^{2}$ & $\mathrm{Indiv} / \mathrm{m}^{2}$ & $\mathrm{~g} / \mathrm{m}^{2}$ & Indiv $/ \mathrm{m}^{2}$ & $\mathrm{~g} / \mathrm{m}^{2}$ \\
\hline $901-1000$ & & & 1.46 & 22.91 & & \\
$801-900$ & & & 1.24 & 22.51 & & \\
$701-800$ & & & 1.48 & 21.39 & & \\
$601-700$ & & & 1.93 & 35.01 & & \\
$501-600$ & 0.16 & 4.98 & 1.21 & 15.97 & & \\
$401-500$ & 0.30 & 5.59 & 0.77 & 8.66 & & \\
$301-400$ & 0.27 & 3.82 & 0.79 & 7.32 & 0.80 & 10.76 \\
$201-400$ & 0.44 & 7.79 & 0.94 & 8.46 & 1.16 & 10.80 \\
$101-200$ & 0.68 & 8.16 & 2.09 & 16.23 & 1.38 & 11.17 \\
$0-100$ & 1.08 & 8.47 & 1.47 & 8.57 & 1.08 & 6.87 \\
\hline Promedio & 0.49 & 6.32 & 1.34 & 16.70 & 1.11 & 9.90 \\
ponderado & & & & & & \\
\hline
\end{tabular}

${ }^{1}$ Octubre (río Ocoña), diciembre (río Majes-Camaná), setiembre (río Tambo)

Cuadro 7. Evolución de los índices de concentración de camarón Cryphiops caementarius en los ríos de la costa sur

\begin{tabular}{lcccccc}
\hline \multirow{2}{*}{ Año } & \multicolumn{2}{c}{ Río Ocoña } & \multicolumn{2}{c}{ Río Majes-Camaná } & \multicolumn{2}{c}{ Río Tambo } \\
\cline { 2 - 7 } & Indiv $/ \mathrm{m}^{2}$ & $\mathrm{~g} / \mathrm{m}^{2}$ & Indiv $/ \mathrm{m}^{2}$ & $\mathrm{~g} / \mathrm{m}^{2}$ & Indiv $/ \mathrm{m}^{2}$ & $\mathrm{~g} / \mathrm{m}^{2}$ \\
\hline 1996 & 0.76 & 3.18 & 0.57 & 3.71 & 0.65 & 3.69 \\
1997 & 1.57 & 9.77 & 0.68 & 6.52 & 0.93 & 8.38 \\
1998 & 0.76 & 4.44 & 0.32 & 3.72 & 0.21 & 1.58 \\
1999 & 0.70 & 10.52 & 0.69 & 6.99 & 0.94 & 5.31 \\
2000 & 1.39 & 18.31 & 0.80 & 11.00 & 1.13 & 9.23 \\
2001 & 0.69 & 12.88 & 0.67 & 10.22 & 0.89 & 9.08 \\
2002 & 1.60 & 20.87 & & & & \\
2004 & 1.07 & 13.32 & & & 1.51 & 10.31 \\
2005 & 1.99 & 22.39 & 1.78 & 18.58 & 2.01 & 12.98 \\
2006 & 0.91 & 11.06 & 1.24 & 12.13 & 1.86 & 9.71 \\
2007 & 1.75 & 19.22 & 1.87 & 21.51 & 1.19 & 8.00 \\
2008 & 1.38 & 18.02 & 2.11 & 18.01 & 1.00 & 7.20 \\
2009 & 1.32 & 17.55 & 1.44 & 15.05 & 0.81 & 8.12 \\
2010 & 1.02 & 9.43 & 1.89 & 16.54 & 0.86 & 6.14 \\
2011 & 0.71 & 9.16 & 1.04 & 11.34 & 0.48 & 4.56 \\
2012 & 0.30 & 3.89 & 0.58 & 7.66 & 0.76 & 6.00 \\
2013 & 0.49 & 6.32 & 1.34 & 16.70 & 1.11 & 9.90 \\
\hline
\end{tabular}

${ }^{1}$ Fuente: Prospecciones de evaluación poblacional del cama rón de río ejecutadas por IMARPE 
Cuadro 8. Abundancia (N. ${ }^{\circ}$ y biomasa absoluta (kg) de camarón Cryphiops caementarius, según su longitud total $(\mathrm{mm})$, en los ríos de la costa sur (setiembre-diciembre, 2013)

\begin{tabular}{|c|c|c|c|c|c|c|}
\hline \multirow{2}{*}{$\begin{array}{l}\text { Talla } \\
(\mathrm{mm})\end{array}$} & \multicolumn{2}{|c|}{ Rio Ocoña } & \multicolumn{2}{|c|}{ Río Majes-Camaná } & \multicolumn{2}{|c|}{ Río Tambo } \\
\hline & N. ${ }^{\circ}$ & $\mathrm{kg}$ & N. ${ }^{\circ}$ & $\mathrm{kg}$ & N. ${ }^{\circ}$ & $\mathrm{kg}$ \\
\hline \multicolumn{7}{|l|}{$20-25$} \\
\hline \multicolumn{7}{|l|}{$25-30$} \\
\hline $30-35$ & & & & & 4,391 & 4 \\
\hline $35-40$ & 6,450 & 9 & & & 6,586 & 8 \\
\hline $40-45$ & 12,901 & 22 & 4,546 & 6 & 24,149 & 46 \\
\hline $45-50$ & 53,754 & 145 & 145,475 & 335 & 63,666 & 185 \\
\hline $50-55$ & 139,761 & 492 & 413,695 & 1,307 & 217,341 & 823 \\
\hline $55-60$ & 251,569 & 1,118 & 672,823 & 2,780 & 329,305 & 1,154 \\
\hline $60-65$ & 374,128 & 2,153 & 954,681 & 4,959 & 395,165 & 2,395 \\
\hline $65-70$ & 393,480 & 2,878 & 877,397 & 5,718 & 289,788 & 2,156 \\
\hline $70-75$ & 425,732 & 3,788 & $1^{\prime} 086,518$ & 8,893 & 232,709 & 2,148 \\
\hline $75-80$ & 348,327 & 3,833 & 1'109,248 & 10,895 & 144,894 & 1,698 \\
\hline $80-85$ & 268,770 & 3,643 & $1^{\prime} 004,688$ & 12,081 & 90,010 & 1,385 \\
\hline $85-90$ & 187,064 & 3,252 & 813,752 & 11,565 & 65,861 & 1,208 \\
\hline $90-95$ & 133,310 & 2,800 & 545,532 & 9,621 & 43,907 & 969 \\
\hline $95-100$ & 77,406 & 1,967 & 245,489 & 5,165 & 37,321 & 933 \\
\hline $100-105$ & 51,604 & 1,529 & 154,567 & 4,320 & 24,149 & 685 \\
\hline $105-110$ & 51,604 & 1,755 & 90,922 & 3,081 & 10,977 & 360 \\
\hline $110-115$ & 43,003 & 1,768 & 77,284 & 3,216 & 10,977 & 460 \\
\hline $115-120$ & 17,201 & 781 & 77,284 & 3,789 & 2,195 & 103 \\
\hline $120-125$ & 21,502 & 1,177 & 81,830 & 4,098 & & \\
\hline $125-130$ & 10,751 & 648 & 36,369 & 2,212 & & \\
\hline $130-135$ & 4,300 & 362 & 31,823 & 2,051 & & \\
\hline $135-140$ & 2,150 & 180 & 18,184 & 1,299 & & \\
\hline $140-145$ & & & 4,546 & 349 & & \\
\hline $145-150$ & & & 4,546 & 402 & & \\
\hline $150-155$ & & & 4,546 & 439 & & \\
\hline \multicolumn{7}{|l|}{$155-160$} \\
\hline Total & $2^{\prime} 874,769$ & 34,299 & $8^{\prime} 455,747$ & 98,580 & $1^{\prime} 993,390$ & 17,120 \\
\hline
\end{tabular}

Abundancia y biomasa

La abundancia (número de ejemplares) y biomasa $(\mathrm{kg})$ fue dependiente del estrato altitudinal y de la talla del camarón.

En el río Ocoña, la abundancia del recurso estuvo mayoritariamente concentrada en el estrato de 0 a $100 \mathrm{msnm}$, conformado por ejemplares de 65 a $80 \mathrm{~mm}$ de longitud total. En el río Majes-Camaná, la concentración fue mayor en el estrato de 101 a $200 \mathrm{msnm}$, compuesto por ejemplares de mayor talla (70 a $85 \mathrm{~mm}$ ). Asimismo, en el río Tambo el recurso se concentró en el estrato de 101 a 200 msnm, compuesto por ejemplares de 55 a 70 $\mathrm{mm}$ de longitud total (Cuadros 8 y 9). 
Cuadro 9. Abundancia $\left(\mathrm{N} .^{\circ}\right)$ y biomasa absoluta $(\mathrm{kg})$ de camarón Cryphiops caementarius, según el estrato altitudinal, en los ríos de la costa sur (setiembre-diciembre, 2013)

\begin{tabular}{lcccccc}
\hline \multirow{2}{*}{$\begin{array}{l}\text { Estratos } \\
(\mathrm{msnm})\end{array}$} & \multicolumn{2}{c}{ Río Ocoña } & \multicolumn{2}{c}{ Río Majes-Camaná $^{2}$ Río Tambo } \\
\cline { 2 - 7 } $\mathrm{N}^{\circ}$ & $\mathrm{kg}$ & $\mathrm{N}^{\circ}$ & $\mathrm{kg}$ & $\mathrm{N} .^{\circ}$ & $\mathrm{kg}$ \\
\hline $901-1000$ & & & 575,236 & 9,054 & & \\
$801-900$ & & & 298,197 & 5,433 & & \\
$701-800$ & & & $1^{\prime} 707,681$ & 24,714 & & \\
$601-700$ & & & 967,258 & 17,555 & & \\
$501-600$ & 123,278 & 3,179 & 584,081 & 7,703 & & \\
$401-500$ & 286,872 & 5,292 & 451,574 & 5,057 & & \\
$301-400$ & 194,917 & 2,797 & 365,332 & 3,382 & 254,343 & 3,423 \\
$201-400$ & 315,592 & 5,574 & 591,887 & 5,307 & 437,725 & 4,070 \\
$101-200$ & 519,713 & 6,256 & $1^{\prime} 765,198$ & 13,677 & 785,998 & 6,357 \\
$0-100$ & $1^{\prime} 434,397$ & 11,201 & $1^{\prime} 149,303$ & 6,698 & 515,324 & 3,270 \\
\hline Total & $2 ' 874,769$ & 34,299 & $8^{\prime} 455,747$ & 98,580 & $1 ' 193,390$ & 17,120 \\
\hline
\end{tabular}

Cuadro 10. Evolución de la abundancia (individuos $\left./ \mathrm{m}^{2}\right)$ y biomasa absoluta $\left(\mathrm{g} / \mathrm{m}^{2}\right) \mathrm{de}$ camarón en los ríos de la costa sur

\begin{tabular}{|c|c|c|c|c|c|c|}
\hline \multirow{2}{*}{ Año } & \multicolumn{2}{|c|}{ Río Ocoña } & \multicolumn{2}{|c|}{ Río Majes-Camaná } & \multicolumn{2}{|c|}{ Río Tambo } \\
\hline & Individuos & $\mathrm{kg}$ & Individuos & $\mathrm{kg}$ & Individuos & $\mathrm{kg}$ \\
\hline 2005 & $11^{\prime} 394,108$ & 127,919 & $10^{\prime} 953,312$ & 114,382 & $4^{\prime} 007,100$ & 25,918 \\
\hline 2006 & $5 ’ 245,188$ & 63,892 & $5,585,960$ & 54,541 & $2 ’ 546,294$ & 13,271 \\
\hline 2007 & 9'440,404 & 103,829 & $8^{\prime} 639,902$ & 99,326 & $2 ’ 140,678$ & 14,437 \\
\hline 2008 & 6’403,948 & 83,448 & $4^{\prime} 794,682$ & 40,911 & $1 ' 159,345$ & 8,333 \\
\hline 2009 & $5,383,916$ & 71,569 & 5'440,697 & 56,794 & 936,533 & 9,366 \\
\hline 2010 & $5^{\prime} 237,353$ & 48,336 & $9 ’ 476,535$ & 82,754 & $1^{\prime} 188,254$ & 8,459 \\
\hline 2011 & 3'010,326 & 37,031 & $5 ’ 430,685$ & 59,432 & 557,078 & 5,332 \\
\hline 2012 & 1'954,432 & 22,765 & 3'930,733 & 48,230 & $1 ’ 346,832$ & 9,960 \\
\hline 2013 & $2^{\prime} 874,769$ & 34,299 & $8^{\prime} 455,747$ & 98,580 & 1’993,390 & 17,120 \\
\hline
\end{tabular}

En relación con la biomasa, en el río Ocoña el recurso presentó la mayor biomasa en el estrato de 0 a $100 \mathrm{msnm}$, mientras que en los ríos Majes-Camaná y Tambo se presentaron en el estrato de 101 a $200 \mathrm{msnm}$ (Cuadro 9). Estos resultados indican que el río Majes-Camaná presentó la mayor abundancia de camarón y la mayor biomasa en el
2013 (el 63.5 y $65.7 \%$ del total de los tres ríos, respectivamente).

La evolución anual de la abundancia y biomasa absoluta de camarón Los datos acumulados hasta el 2012 para los ríos en estudio, indican una tendencia negativa en los valores de densidad (Cuadro 10). En 2012 se observó una situación poblacional crítica del 
recurso, principalmente en el caso de los ríos Ocoña y Majes-Camaná, registrándose los más bajos índices de concentración de la última década (Cuadro 10). Esta delicada situación sería el corolario de la sobrepesca que afecta en mayor medida a los ejemplares más grandes, la presión de pesca ejercida en particular sobre las hembras en los estratos altitudinales inferiores, así como de la pesca ilegal y otros factores de origen antrópico (Monitoreos poblacionales de camarón de río 2011, 2012 y 2013 ejecutados por IMARPE).

No obstante, en las evaluaciones poblacionales del presente estudio, se apreciaron ciertos incrementos en los índices de concentración en estos ríos, especialmente en el río Majes-Camaná. Es de esperarse que estos resultados sean indicios de una recuperación poblacional moderada del recurso, el cual podría obedecer a factores tales como una mayor disponibilidad del recurso hídrico durante el año, así como a una mayor atención en la aplicación de las medidas normativas de protección del recurso.

\section{Conclusiones}

- Considerando la serie histórica de los índices poblacionales anuales de concentración de camarón, hasta el 2012 se observó una tendencia negativa de los valores en los tres ríos.

- En 2012 se apreció una situación poblacional crítica del recurso, principalmente en el caso de los ríos Ocoña y Majes-Camaná, donde se registraron los más bajos índices de concentración de la última década.

- En el 2013 los ríos Tambo, Ocoña y Majes-Camaná muestran incrementos en los índices de concentración, siendo más evidente en el río Majes-Camaná. La ratificación de esta tendencia favorable para el recurso podrá apreciarse mejor en posteriores evaluaciones.

\section{Literatura Citada}

1. Amaya de GJ, Guerra A. 1976. Especies de camarones de los ríos norteños del Perú y su distribución. Informe del Convenio Ministerio de Pesquería - Univ Trujillo. Ministerio de Pesquería, Lima, Perú. 58 p.

2. Ancieta F. 1950. El camarón de río. Pesca y caza. Lima, Perú. 7 p.

3. Báez P. 1985. Fenómeno El Niño, elemento importante en la evolución del camarón de río (Cryphiops caementarius). Inv Pesq 32: 235-242.

4. Bahamonde N, Vila I. 1971. Sinopsis sobre la biología del camarón de río del norte. Biol Pesq 5: 3-60.

5. Castro C. 1966. El camarón de río del norte Cryphiops caementarius (Molina). Est Oceanol 2: 11-19.

6. Chávez $R$, De Parodi E, Villegas J. 1973. Estudio del Cryphiops caementarius (Molina) (camarón de río). Rev Inv Univ Nac San Agustín 2(1): 13-34.

7. Elías J. 1960. Contribución al comportamiento del camarón de río Cryphiops caementarius (Molina) Decapoda: Palaemonidae. Pesca y caza 10: 84-106.

8. Espino M, Wosnitza-Mendo C. 1984. Manuales de evaluación de peces N. ${ }^{\circ}$ 1. Área barrida. Inf Instituto del Mar del Perú N. ${ }^{\circ}$ 86. Lima: IMARPE. 32 p.

9. Hartmann $G$ 1958. Apuntes sobre la biología del camarón de río, Cryphiops caementarius (Molina) Palaemonida, Decapada. Rev. Pesca y caza 8: 15-28.

10. Holthuis LB. 1952. A general revision of the Palaemonidae (Crustacea Decapoda Natantia) of the Americas. II. The subfamily Palaemonidae. California, USA: Univ South California. 396 p.

11. Meruane J, Rivera M, Morales C, Galleguillos C, Hosokawa H. 2006. Juvenile production of the freshwater prawn Cryphiops caementarius (Decapoda: Palaemonidae) under laboratory conditions in Coquimbo, Chile. Gayana 70: 228-236. 
12. Munaylla U. 1977. Desarrollo larval del «camarón de río» Cryphiops caementarius (Molina, 1782): determinación y descripción de sus estadios larvarios. Documenta 62: 12-16.

13. Norambuena R. 1997. Antecedentes biológicos de Cryphiops caementarius (Molina, 1782) en el estero «El Culebrín» (Crustacea, Decapoda, Palaemonidae). Biol Pesq 9: 7-19.

14. Rivera M, Schmiede P, Meruane J. 1983. Desarrollo larval del camarón de río del norte Cryphiops caementarius (Molina, 1782) (Crustacea: Palaemonidae), en condiciones de laboratorio. En: Symposio Internacional de Acuacultura. Coquimbo, Chile. p 315-334.

15. Sansana J, Baez P. 1983. Desarrollo larvario de Cryphiops caementarius (Molina, 1782) en condiciones de laboratorio (Crustacea: Decapoda: Palaemonidae). En Mem Asoc Latinoam Acuicult 5: 347-353.
16. Vegas M, Ruiz LA, Sanchez S. 1981. El camarón Cryphiops caementarius (Palaemonidae): desarrollo embriológico, contenido estomacal y reproducción controlada: primeros resultados. Rev Latinoam Acuicult 9: 11-28.

17. Viacava M, Aitken R, Llanos J. 1978. Estudio del camarón en el Perú. Bol Inst Mar del Perú 3(5): 165-232.

18. Yavar C, Dupre. 2007. Desarrollo embrionario del camarón de río Cryphiops caementarius (Decapoda: Palaemonidae) en condiciones de laboratorio. Biol Trop 55(Supl1): 15-24.

19. Yépez V. 2009. Consideraciones acerca de la distribución y extracción del recurso «camarón» en ríos de la costa Peruana. Pesca 101(7-8): 9-11.

20. Yépez, V, Bandin R. 1996. Estimación poblacional del camarón Cryphiops caeme ntarius Molina 1782 (Natantia, Palaemonidae) en los ríos Ocoña, Majes Camaná y Tambo. Inf Prog Instituto del Mar del Perú 43: 3-31. 